\title{
Melhorando o desempenho de um subsistema móvel de monitoramento de subestações de energia modelado a partir de um Web Service
}

\author{
Martin Dill ${ }^{1}$, Sérgio Dill ${ }^{1}$, Edson Padoin ${ }^{1}$, Paulo Sausen ${ }^{1}$ e Airam Sausen ${ }^{1}$
}

\begin{abstract}
Resumo: Neste artigo é apresentada uma metodologia de substituição de tecnologias e ferramentas em um subsistema móvel de monitoramento de subestações de energia elétrica objetivando a melhoria do desempenho do mesmo. Com a inclusão de melhorias no subsistema a partir da adição de novos módulos e, principalmente, da substituição do Web Service, foi possível melhorar o desempenho, a segurança e a confiabilidade do sistema como um todo.
\end{abstract}

Palavras-chave: Sistema de Automação. Web Service. Maemo.

\begin{abstract}
This article presents a methodology for replacement of tools and technologies in a mobile subsystem monitoring electrical substations designed to improve their performance. With the inclusion of improvements in the subsystem from the addition of new modules and especially replacement of Web Service could improve performance, safety and reliability of the system as a whole.
\end{abstract}

Keywords: Automation Systems. Web Service. Maemo.

\section{Introdução}

A Computação Móvel e Ubíqua está sendo considerada a nova era da computação, pois representa uma mudança de paradigma da era atual, marcada pelos sistemas centrados em computadores desktop, para uma nova era, centrada na ubiquidade das comunicações e dos dispositivos móveis. Tais dispositivos permitem ao usuário final o fácil acesso e processamento da informação das mais variadas tarefas do dia a dia, a qualquer momento, em qualquer lugar, a partir de qualquer dispositivo.

A crescente popularidade dos dispositivos móveis, capazes de se conectar a internet através de redes sem fio (e.g., Internet Tablets), tem criado a necessidade da disponibilização de novas aplicações que possam ser executadas nesses dispositivos, as quais se diferenciam das aplicações tradicionais, que são executadas nos desktops, em decorrência das limitações de hardware e de software.

Por se tratar de um mercado relativamente novo, a demanda por profissionais capacitados para desenvolver essas aplicações é alta, e a quantidade de informações relacionadas com o desenvolvimento de aplicações para os dispositivos móveis ainda é escassa e mal estruturada. Um exemplo de segmento que está em alta no mercado atualmente são os Internet Tablets (e.g., Nokia N900), que executam as suas aplicações sob a plataforma maemo.

\footnotetext{
${ }^{1}$ Lacop - Laboratório de Computação Pervasiva, GAIC - Grupo de Automação Industrial e Controle, DeTEC - Departamento de Tecnologia, UNIJUI - Universidade Regional do Noroeste do Estado do Rio Grande do Sul, Rua Lulu Ilgenfritz 480, Bairro São Geraldo, Ijuí - RS

\{martin.dill, dill, padoin, sausen,airam\}@unijui.edu.br

doi: $10.5335 /$ rbca. 2011.007
}

Revista Brasileira de Computação Aplicada (ISSN 2176-6649), Passo Fundo, v. 3, n. 1, p. 64-73, mar. 201164 
Este artigo é uma extensão de Dill (2009) e apresenta a evolução de um sistema de monitoramento a partir do aperfeiçoamento e substituição de tecnologias e ferramentas em um subsistema de computação móvel aplicado ao monitoramento de subestações de energia elétrica, que é parte integrante de um Sistema de Automação de Subestações. Esse subsistema foi desenvolvido sob a plataforma maemo e foi construído sobre um framework de referência estruturado totalmente sobre tecnologias livres de custo, com o objetivo de auxiliar desenvolvedores no estágio inicial de desenvolvimento de aplicações para esta plataforma.

O restante deste artigo está organizado da seguinte forma: na seção 2 é apresentado, brevemente, o Sistema de Automação de Subestações, e também o subsistema móvel de monitoramento; na sequência, na Seção 3, as inovações aplicadas ao subsistema móvel são descritas e detalhadas; finalmente, na Seção 4 são apresentadas as considerações finais do artigo.

\section{Sistema de monitoramento de subestações de energia e1létrica}

O Sistema de Monitoramento de Subestações de Energia Elétrica foi desenvolvido pelo Grupo de Automação Industrial e Controle (GAIC) da Unijuí. Este sistema utiliza o conceito de sensores inteligentes estruturados a partir de uma Rede de Sensores Sem Fio (RSSF) e foi totalmente desenvolvido sobre plataforma de software livre, unindo num mesmo sistema de supervisão os conceitos de sensores inteligentes, mobilidade, software livre e baixo custo. Na Figura 1 é apresentada a arquitetura deste sistema; mais detalhes sobre o mesmo podem ser encontrados em Salvadori (2003), Sausen (2007) e Salvadori (2009).

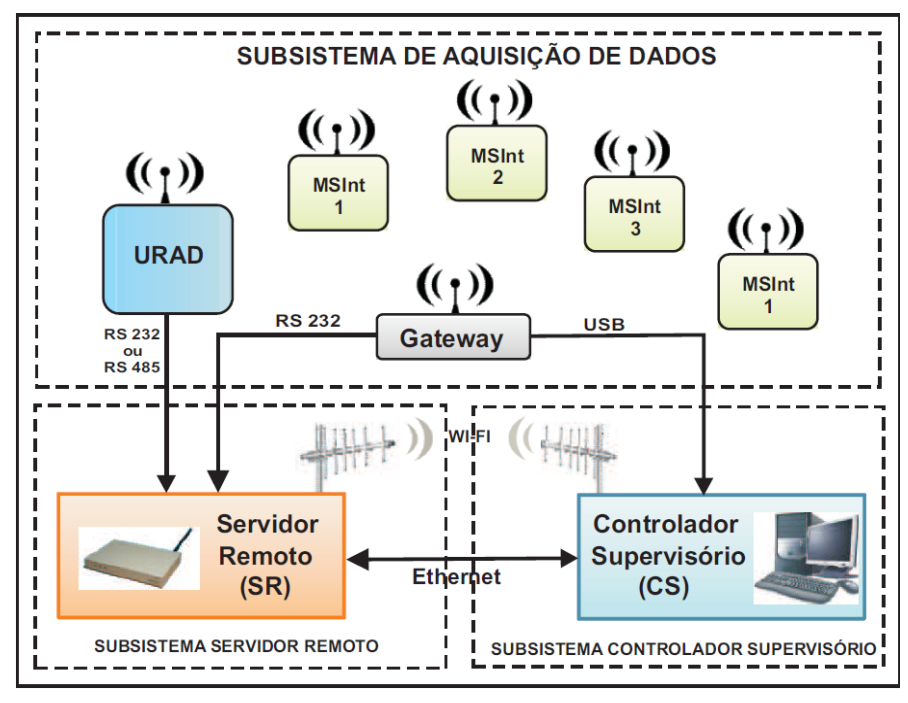

Figura 1. Arquitetura do sistema de monitoramento de subestações.

O Sistema de Monitoramento de Subestações é subdividido em três subsistemas: o Subsistema de Aquisição de Dados, o Subsistema Servidor Remoto e o Subsistema Controlador Supervisor.

O Subsistema de Aquisição de Dados é formado por uma Unidade Remota de Aquisição de Dados (URAD) e pelo Módulo de Sensores Inteligentes (MSInt), que são dispositivos que coletam os dados de uma linha de transmissão de energia elétrica. Os dados coletados pela URAD (i.e., Valores de Tensão, Corrente e Potência em um sistema trifásico) são transmitidos para o Subsistema Servidor Remoto (SR), por meio de uma conexão wireless, e para o Subsistema Controlador Supervisor (CS), via interface RS 232, conforme pode ser observado na Figura 1.

As funções necessárias para o Servidor Remoto no Subsistema Servidor Remoto são disponibilizadas a partir da utilização de uma Soekris modelo Net4521. Este hardware é apropriado para operar em lugares inóspitos sob severas condições de funcionamento. O Sistema Operacional utilizado na Soekris é o Voyage Linux, uma distribuição Linux baseada em Debian e customizada para trabalhar em plataformas voltadas para sistemas embarcados. Mais detalhes sobre o módulo Soekris e sua implementação no sistema de monitoramento de subestações podem ser obtidos em Padoin (2007). 
O Subsistema Controlador Supervisor (CS), desenvolvido em linguagem Java, é responsável pela exibição das grandezas coletadas pelo subsistema de Aquisição de Dados em gráficos. Os valores numéricos das grandezas, juntamente com a data e hora, são aquisitados a cada 16 milissegundos e armazenados no Sistema de Gerenciamento de Banco de Dados (SGBD). Além do monitoramento, o subsistema CS dispara alarmes, que são ativados no momento em que são ultrapassados os limites previamente configurados nos parâmetros do aplicativo, como, por exemplo, sub e sobre tensão.

\subsection{Subsistema de monitoramento móvel}

A partir do Sistema de Monitoramento de Subestações apresentado em Salvadori (2009), foi desenvolvido um subsistema, que consiste em um módulo móvel, independente e remoto do Sistema de Monitoramento de Subestações de Energia Elétrica. O Subsistema de monitoramento móvel de subestações de energia elétrica foi desenvolvido em três níveis, o primeiro é o SGBD, que possui a base de dados onde são armazenadas todas as grandezas coletadas pela URAD; o segundo é o Web Service (WS), que contém todos os métodos necessários para a leitura dos dados na base e a linguagem para comunicar-se com uma aplicação e enviar os dados para a mesma; o terceiro é composto por uma aplicação, que o usuário utilizará para monitorar a subestação de energia a partir de um dispositivo móvel.

\subsubsection{Base de dados}

A utilização de um SGBD, hoje em dia, é essencial para o desenvolvimento de sistemas, porque propicia muitos benefícios, entre os quais: maior velocidade na recuperação de dados, grande capacidade de indexação, controle de inconsistência dos dados, maior segurança e múltiplas transações (pode-se ler e gravar no banco de dados ao mesmo tempo). Todas essas características não estão presentes em um sistema de arquivos tradicional. Neste caso, como se trata de uma aplicação em tempo real, as gravações no banco de dados devem ser rápidas, para que o usuário não receba dados antigos na aplicação Navathe (2002).

O SGBD utilizado no sistema de monitoramento de subestações é o IBM DB2 Express-C. Na base de dados há uma tabela principal que guarda todos os dados aquisitados pela URAD e, por meio desta base de dados, o WS consegue recuperar todos os dados necessários para a aplicação.

\subsubsection{Web Service}

O WS é o nível que se situa entre a base de dados e a aplicação, uma solução utilizada na integração de sistemas e na comunicação entre aplicações diferentes. A partir dele é possível que novas aplicações possam interagir com aquelas que já existem e que sistemas desenvolvidos em plataformas diferentes sejam compatíveis. É a situação existente neste subsistema quando é necessário incorporar este subsistema ao sistema de monitoramento de subestação já existente.

Neste nível houve uma mudança significativa, pois foi necessária a substituição do WS desenvolvido na linguagem de programação Python por um software mais robusto desenvolvido em Java (mais detalhes na seção $3)$.

\subsubsection{Aplicação}

A aplicação de monitoramento móvel de subestações foi projetada para ser executada nos dispositivos móveis da família dos Internet Tablets da Nokia (e.g. N800, N900), utilizando os conceitos de um framework de referência para a plataforma maemo/Symbian (plataforma dos Internet Tablets da Nokia), cujo propósito é tornar o desenvolvimento de aplicações para esta plataforma mais simplificado.

Este framework foi construído totalmente a partir da utilização de tecnologias livres de custo, utilizando ferramentas open source (i.e., código aberto), que podem ser instaladas em um computador desktop com sistema operacional Linux, o que torna o desenvolvimento de aplicações mais acessível economicamente, pois não é necessário possuir um dispositivo móvel para testar as aplicações desenvolvidas; esta tarefa pode ser realizada diretamente no ambiente de desenvolvimento. Esta prática também é vantajosa se a equipe de desenvolvedores for numerosa, pois cada um poderá desenvolver suas aplicações no seu próprio ambiente. No caso deste trabalho, o dispositivo móvel Nokia N900 foi utilizado para portar a aplicação. O framework de referência para desenvolvimento de aplicações com ênfase na plataforma maemo/Symbian pode ser visualizado na Figura 2; mais detalhes sobre o framework e as suas ferramentas e tecnologias podem ser encontrados em Dill (2008). 


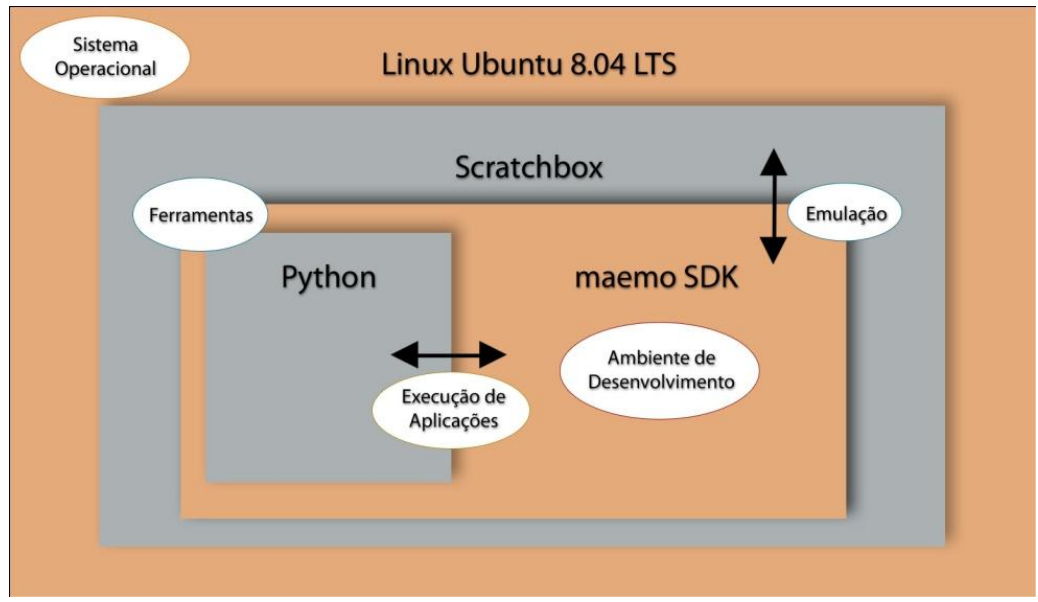

Figura 2. Framework de Referência

A aplicação de monitoramento móvel foi desenvolvida na linguagem de programação Python. O motivo da escolha desta linguagem recai em ser uma linguagem simples de ser declarada e que, ao mesmo tempo, possui recursos poderosos para o desenvolvimento de aplicações, o que facilita o processo de desenvolvimento. A aplicação funciona da seguinte forma: ao executar a aplicação, abre-se a tela do Menu Principal (Figura 3), onde podem ser escolhidas as opções Tensão e Corrente na fase A, B e C, Potência Ativa, Potência Aparente e Fator de Potência.

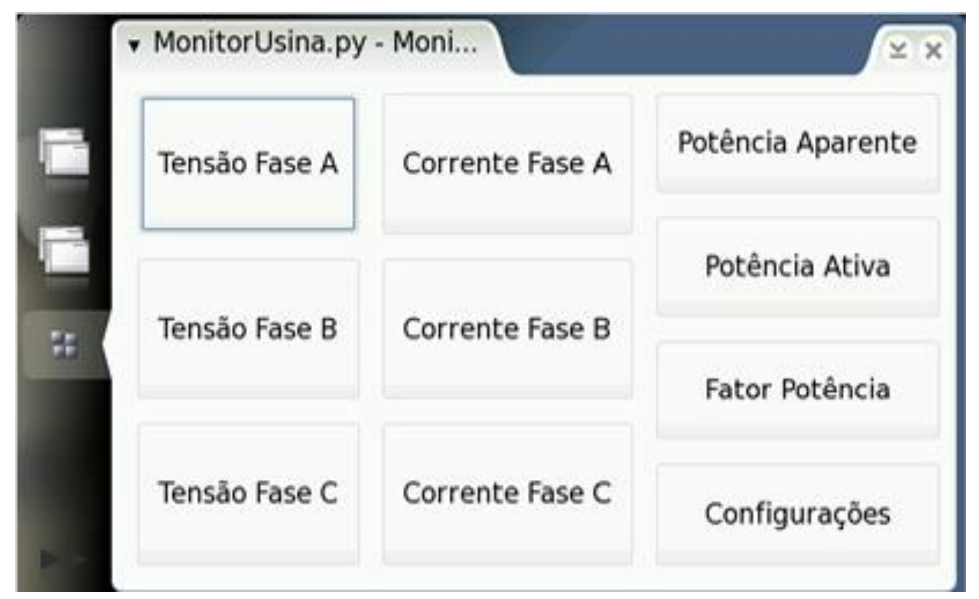

Figura 3. Menu Principal da Aplicação

Ao clicar em uma das telas, abre-se uma nova janela, onde são exibidas, em forma de gráfico, as grandezas coletas pela URAD em tempo real, que são armazenadas na base de dados do servidor. A aplicação conecta ao WS para capturar os dados e o WS realiza o acesso à base de dados, pois não é possível realizar acesso direto ao SGBD pela aplicação. Um exemplo de tela exibindo um gráfico dos valores coletados pode ser visualizado na Figura 4. 


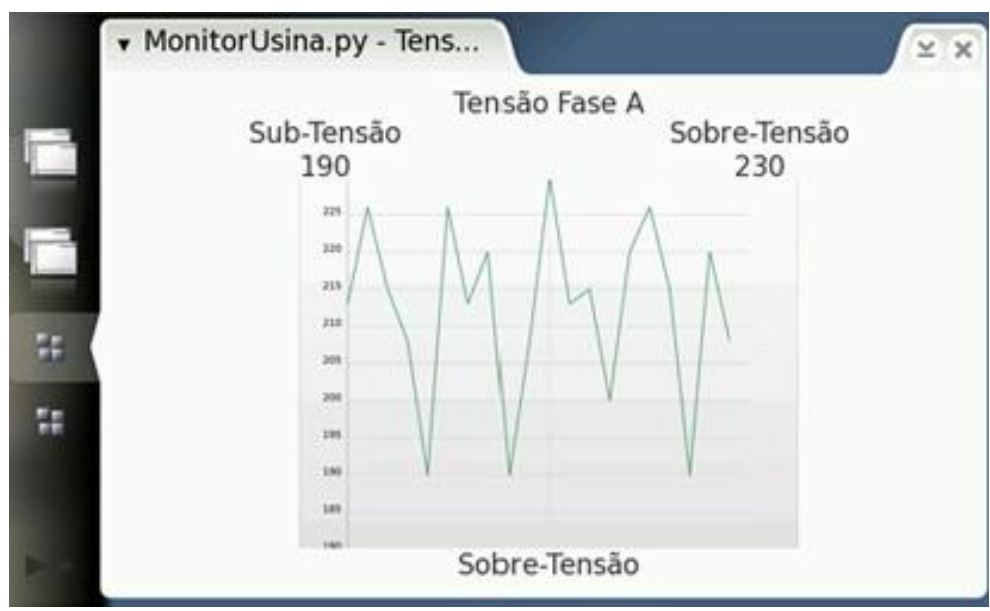

Figura 4. Tela Tensão Fase A

Também é possível editar as configurações (Figura 5), limites das grandezas e configurações da base de dados. Na aba "Limites" é possível configurar a tensão nominal da subestação (Volts), os limites de sub e sobre tensão (\%), o limite de sobrecarga (Amperes) e também o intervalo de tempo (segundos) em que serão atualizados os dados nos gráficos.

Na aba "Banco de dados" é possível alterar o endereço IP do servidor do WS, o nome da base de dados, o usuário e a senha para acessar a base. Essas configurações não são registradas em uma base de dados, por serem poucos bytes de informação; portanto, foi utilizado um arquivo sequencial (arquivo de texto) para armazenar essas configurações, o qual fica salvo no diretório raiz da aplicação.

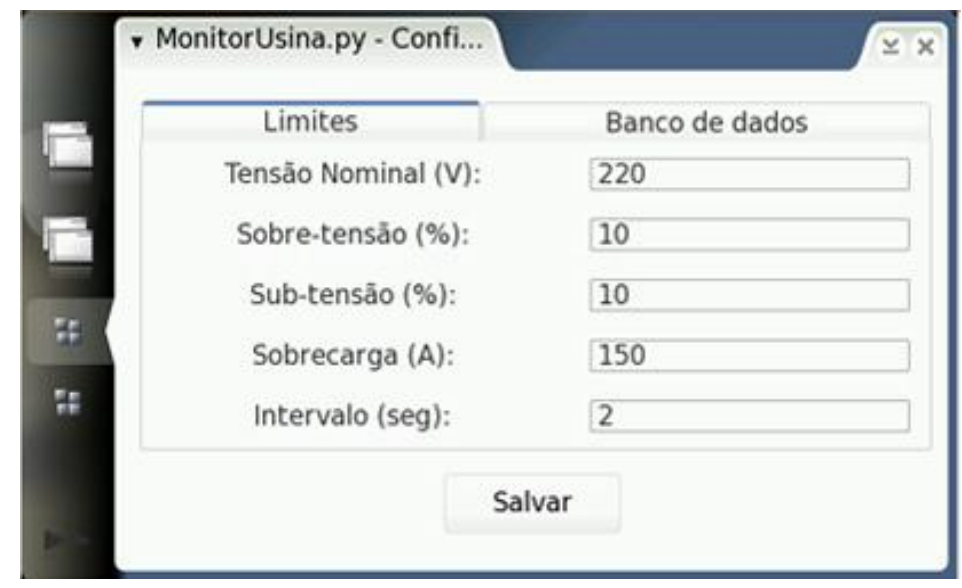

Figura 5. Tela Configurações

O esquema organizacional do Sistema de Monitoramento de Subestações, juntamente com o Subsistema de Monitoramento Móvel, pode ser visualizado na Figura 6. Mais informações sobre o subsistema móvel e suas partes integrantes podem ser acessadas em Dill (2009). 


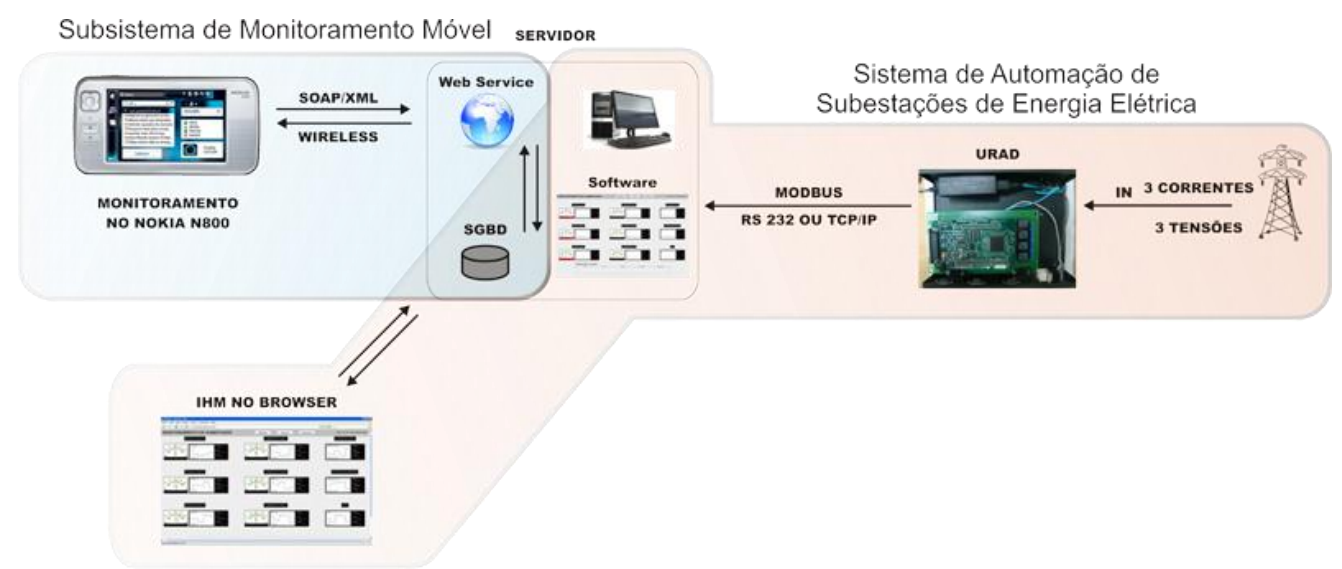

Figura 6. Esquema Organizacional do Sistema

\section{Aperfeiçoamento de aplicação e substituição de ferramentas}

Nesta seção são apresentadas as alterações realizadas na aplicação, que adicionaram novas funções para a realização de consultas em intervalos de tempo, e também para deteção de erros, juntamente com a substituição de tecnologias, que possibilitaram melhor desempenho na recuperação de dados e estabilidade da aplicação.

\subsection{Substituição do Web Service}

Após o desenvolvimento inicial da aplicação de monitoramento de subestações, cujo objetivo era validar o framework para desenvolvimento de aplicações com ênfase na plataforma maemo, apresentado anteriormente, foi constatado, a partir de testes realizados com a aplicação em conjunto com o Web Service, que a mesma possuía um desempenho insatisfatório utilizando o WS inicialmente foi proposto. O WS desenvolvido em Python utilizando o módulo SOAPpy, usado para disponibilização de serviços, apresenta alguns erros ao recuperar dados na base de dados do servidor; também não possuía um tempo de resposta satisfatório para as solicitações da aplicação cliente, tornando o método inaceitável para seu propósito.

O problema encontrado em relação à inconsistência deste WS é em decorrência de a linguagem de programação Python não oferecer extenso suporte à conexão com o SGBD utilizado no sistema de monitoramento, os únicos módulos encontrados para realizar as conexões com a base encontravam-se desatualizados e seus projetos foram descontinuados, impossibilitando um possível aperfeiçoamento no WS e implicando a substituição do mesmo.

Outro motivo para substituir o WS foi que funcionava apenas na plataforma Windows. Como um dos objetivos deste trabalho era apresentar uma solução em software de baixo custo, existia a necessidade de utilização de um servidor com sistema operacional livre de custo e licença; no caso deste trabalho, utilizou-se o sistema operacional Linux. Por todos esses motivos, o WS do servidor foi substituído pela Ferramenta Apache Tomcat versão 6.0.2, juntamente com a ferramenta para criação e gerenciamento de Web Services Apache Axis2, que gerencia Web Services desenvolvidos na linguagem de programação Java.

\subsection{Aperfeiçoamento da aplicação}

Nesta seção serão apresentados os módulos que foram adicionados à aplicação de monitoramento em tempo real, que adicionaram novas funcionalidades ao sistema, permitindo consultas a registros anteriores da subestação e detecção de erros. 


\subsubsection{Módulo de consulta à base}

Após o desenvolvimento dos módulos de monitoramento em tempo real (tensões, correntes e potências), foi desenvolvido um novo módulo, cujo objetivo é realizar consultas para analisar situações anteriores da subestação. Este módulo é de grande utilidade para o operador da subestação, que pode a qualquer momento acessar os erros que porventura ocorreram na subestação.

Ao clicar no botão Consultar Base, na tela principal do programa, uma nova tela é exibida, contendo um formulário (Figura 7) com as seguintes opções: Grandeza, data, hora inicial, hora final e número de registros. Na opção grandeza é selecionada a grandeza que será consultada na base de dados (Tensões, Correntes, Potências); na opção data, deve ser informada a data a ser consultada; na opção hora inicial, o horário em que se iniciará a consulta.

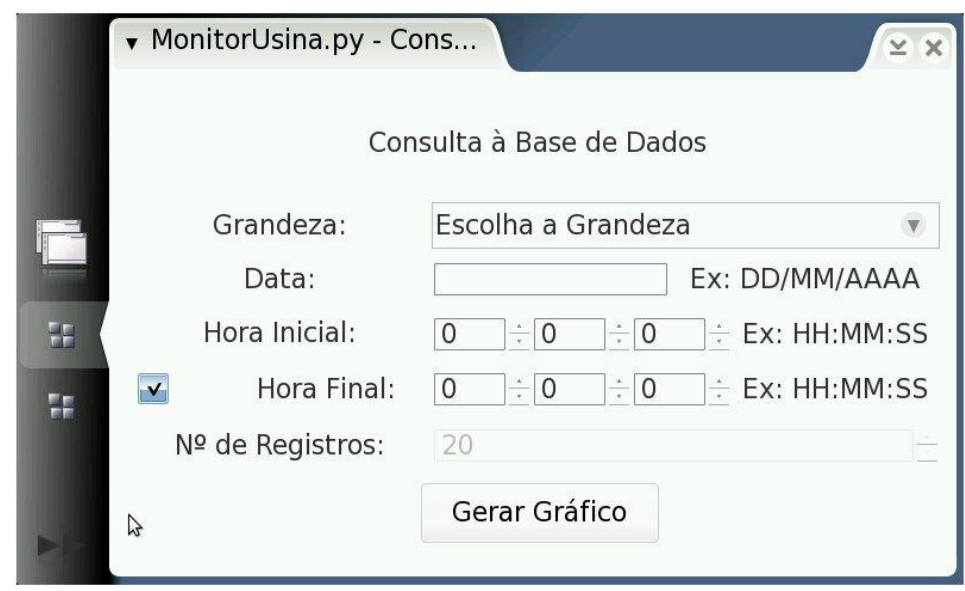

Figura 7. Tela Consulta à Base

Das últimas duas opções, hora final e número de registros, apenas uma pode ser escolhida. Escolhendo a opção hora final, deve-se informar a hora em que terminará a consulta, formando, então, um intervalo de consulta baseado na hora inicial e final. Ao clicar no botão "gerar gráfico", será exibido um gráfico (Figura 8) mostrando a média aritmética de cada minuto no intervalo definido.

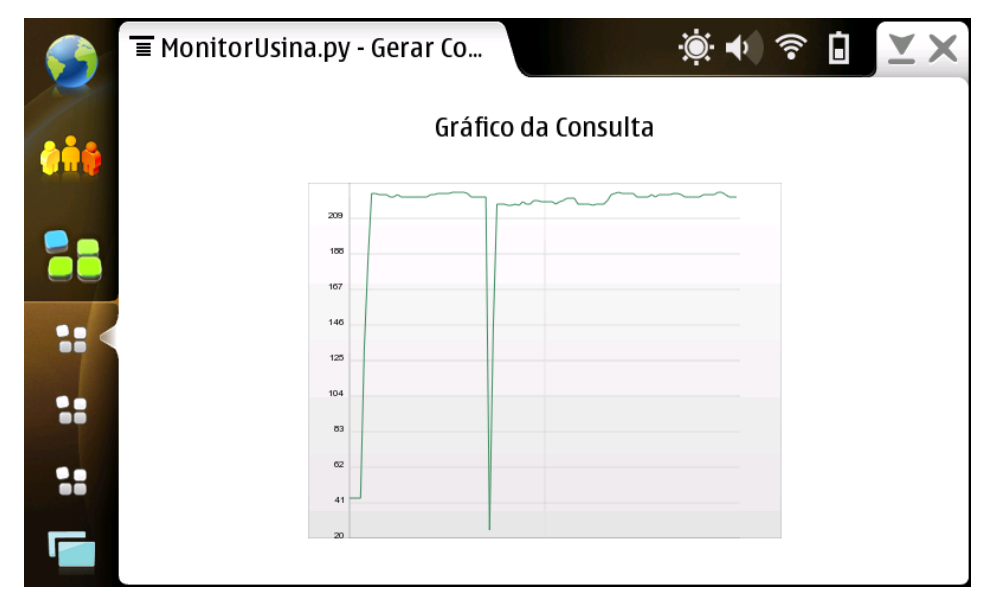

Figura 8. Exemplo de Resultado de Consulta à Base 


\subsubsection{Módulo de Procura de Erros}

Após a conclusão do módulo de consulta à base de dados, foi desenvolvido um novo módulo, que é o módulo de Procura de Erros. Um dos principais objetivos do sistema de monitoramento de subestações é analisar as grandezas coletadas a fim de encontrar erros e, a partir destes erros, localizar as possíveis causas objetivando a melhoria da distribuição de energia para que esses erros não mais ocorram. Este módulo de procura de erros facilita ainda mais essa tarefa.

O módulo de procura de erros visa encontrar as subtensões, sobretensões e sobrecargas ocorridas em um intervalo de tempo definido pelo usuário. Para um dado ser classificado como um erro, deve estar fora da faixa de valores limites definidos na tela de configurações. Ao clicar no botão "Ocorrência de erros", uma nova tela contendo um formulário é carregada (Figura 9), no qual existe a opção "Grandeza", que define a grandeza pela qual serão procurados os erros. Podem ser escolhidas as tensões, correntes ou, até mesmo, todas essas opções.

Ao escolher uma tensão ou uma corrente, deve-se escolher o tipo de erro a ser procurado; os erros possíveis são subtensão e sobretensões, para as tensões, e sobrecarga, para as correntes. Ao escolher a opção "todas", todos os erros serão procurados. As outras três opções - "Data", "Hora Inicial" e "Hora Final" - são referentes ao intervalo de tempo em que serão procurados esses erros.

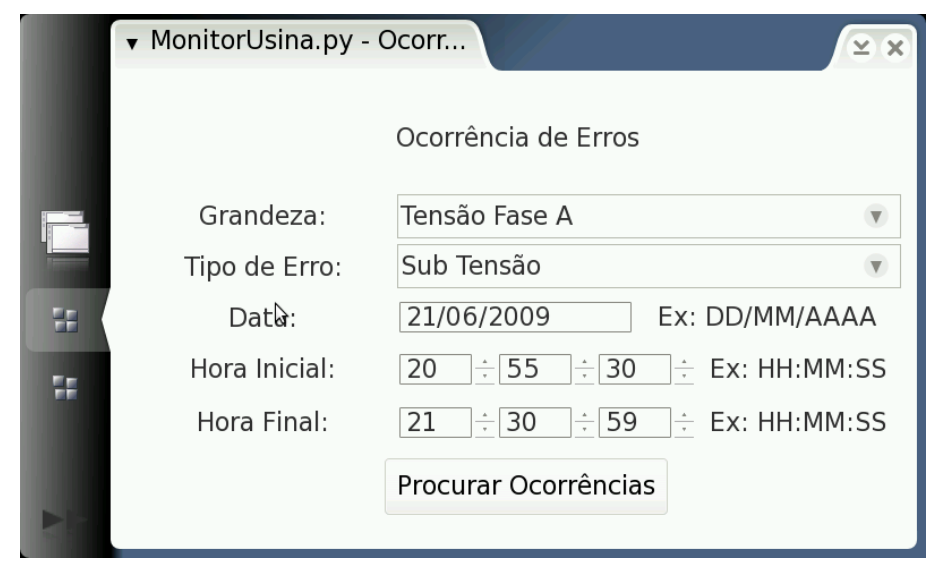

Figura 9. Formulário de Ocorrência de Erros

Ao escolher todas as grandezas, informar o intervalo de tempo e clicar no botão "Procurar Ocorrências", uma requisição é feita para o WS, que procurará na base de dados todos os erros das Tensões e Correntes e exibirá uma nova tela, exibindo os tipos de erros e a quantidade de erros encontrados. Esta tela é apresentada na Figura 10.

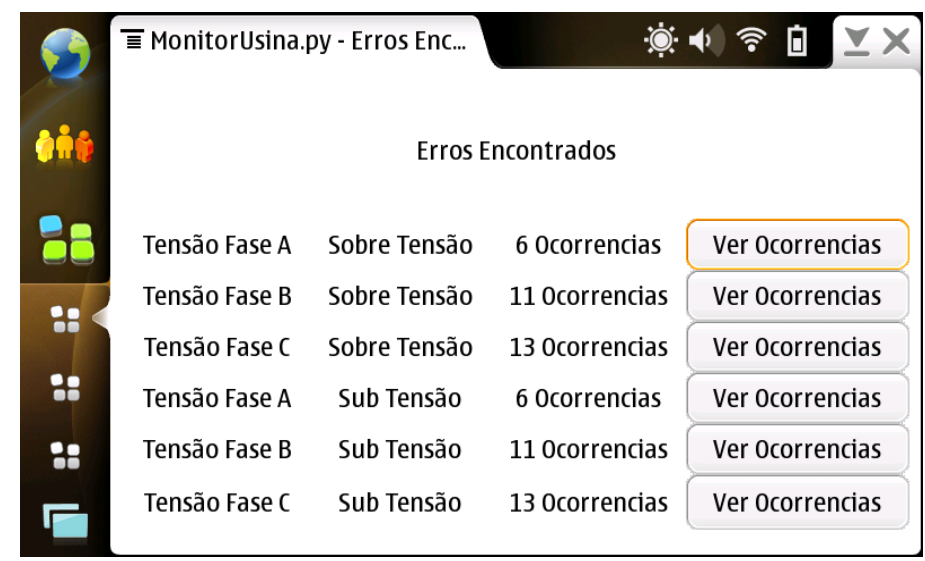

Figura 10. Tela de Erros Encontrados 
Ao clicar em um dos botões de "Ver Ocorrências", serão carregadas as informações sobre os erros em uma nova janela, em formato de tabela. Os erros disponíveis são: Hora inicial do erro, Hora final do erro, Valor Médio (Volts) e Valor do Pico (Volts). Um exemplo desta tela pode ser visualizado na Figura 11.

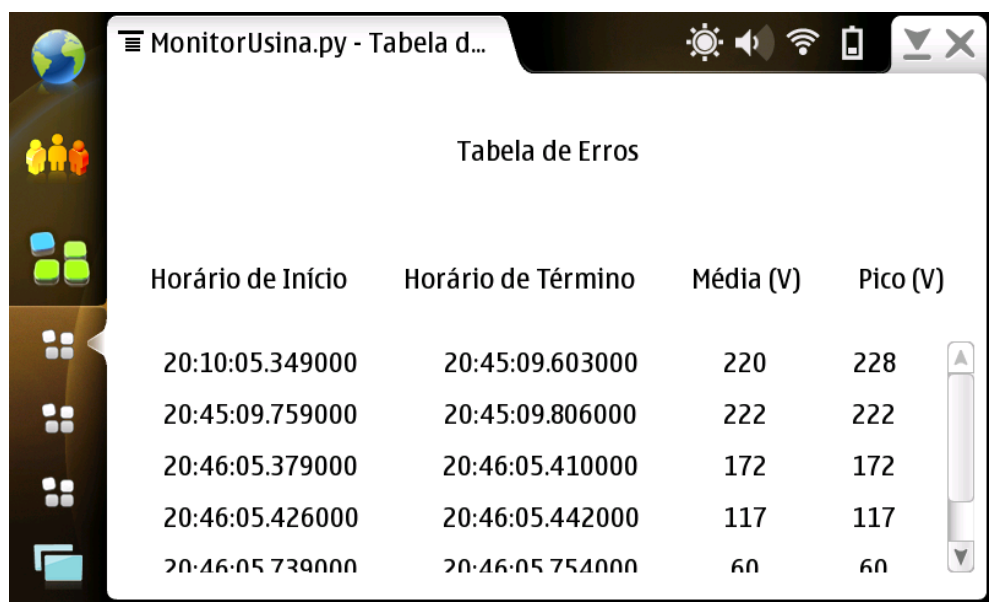

Figura 11. Tabela de informações sobre os erros

Ao escolher apenas uma grandeza para exibição dos erros, a tabela de Erros será exibida de forma direta, pois se trata apenas de uma grandeza e um tipo de erro.

\section{Conclusão}

As substituições realizadas nas tecnologias e ferramentas que formam o subsistema móvel de monitoramento de subestações de energia elétrica proporcionaram melhor desempenho e velocidade na requisição de dados do servidor Web Service para a aplicação cliente. Também propiciaram maior segurança e estabilidade ao sistema, utilizando módulos mais confiáveis e reduzindo, significativamente, a ocorrência de erros de requisição.

As alterações propostas neste artigo tornaram o subsistema apto a realizar a tarefa de monitoramento móvel com maior segurança e confiabilidade, características indispensáveis a tarefa de monitoramento de uma subestação de energia elétrica, que é pouco suscetível a falhas. Outra característica acrescentada ao sistema foi a economia, uma vez que foram utilizadas apenas tecnologias livres de licença, o que possibilitou uma significativa redução de custos. A utilização de um Web Service desenvolvido completamente em Java agregou maior portabilidade ao sistema, uma vez que permite que o mesmo seja executado em várias plataformas, sem a necessidade de ser reescrito ou mesmo compilado.

O desenvolvimento dos módulos de consulta à base de dados e de detecção de erros proporciou a possibilidade de consultas a partir de um intervalo de tempo, permitindo, dessa forma, uma melhor análise dos dados coletados e, por conseguinte, uma melhora significativa na qualidade do monitoramento da subestação de energia elétrica.

\section{Agradecimentos}

Os autores agradecem o apoio financeiro da Fundação de Amparo a Pesquisa do Estado do Rio Grande do Sul (FAPERGS), referente ao Edital 03/2009 - ARD e ao Conselho Nacional de Desenvolvimento Cientifico e Tecnológico (CNPq) pela Bolsa de Iniciação Cientifica e de Produtividade em Pesquisa.

\section{Referências Bibliográficas}

DILL, M. et al. Automação de Subestações de Energia Elétrica Utilizando Dispositivos Móveis. In: Brazilian Conference on Dynamics Control and Applications, DINCON-09. Anais..., 2009. 
DILL, M. Framework para Desenvolvimento de Aplicações maemo para Dispositivos Móveis. Ijuí. Relatório Técnico de Atividades Desenvolvidas no Período de 01/10/2008 a 31/08/2009, UNIJUI, 2008. Disponível online em: $<$ http://www2.unijui.edu.br/ maemo/relatorios/DILL-08.pdf $>$

NAVATHE, E. Sistema de Banco de Dados: Fundamentos e Aplicações. 3. ed. LTC, 2002, p. 795.

Padoin, E. L. et al. Mobilidade no Monitoramento de Subestações Elétricas através de Serviços Web. In: VI SIRC - Simpósio de Informática da Região Centro do RS, VI. Anais..., 2007.

Salvadori, F. et al. Acquisition and Transmission Data Monitoring System Applied to Energy Substation. In: IEEE INTERNATIONAL CONFERENCE ON INDUSTRIAL INFORMATICS, INDIN 2003. Proceedings, p. 60-64, 2003.

Salvadori, F. et al. Monitoring in Industrial Systems Using Wireless Sensor Network With Dynamic Power Management. IEEE Transactions on Instrumentation and Measurement, v. 583104, p. 3104-3111, 2009.

Sausen, P. S. et al. Sistema Baseado em Software Livre para Automação de Subestações Utilizando Redes de Sensores Inteligentes. In: SIMPÓSIO DE AUTOMAÇÃO DE SISTEMAS ELÉTRICOS, VII. Anais..., 2007. 